\title{
Influence du lieu d'hospitalisation sur le devenir à court terme des prématurés nés à 34 semaines d'aménorrhée
}

\author{
THESE \\ PRESENTEE POUR L'OBTENTION DU DOCTORAT EN MEDECINE \\ DIPLÔME D'ETAT \\ CHABAUD François \\ Né le 01/05/1983 à Cavaillon \\ THESE SOUTENUE PUBLIQUEMENT \\ A LA FACULTE DE MEDECINE DE GRENOBLE* \\ Le 13 Janvier 2011
}

DEVANT LE JURY COMPOSE DE

Monsieur le Professeur Dominique PLANTAZ, Président de jury

Monsieur le Professeur Thierry DEBILLON, Directeur de thèse

Madame le Professeur Pascale HOFFMANN

Madame le Docteur Valérie BELIN 
A ce pédiatre qu'est mon père. 


\section{Remerciements}

A Monsieur le Professeur P Plantaz, pour l'honneur que vous me faites en acceptant de présider mon jury de thèse, pour votre disponibilité, votre humanité et votre confiance tout au long de mon internat.

A Monsieur le Professeur T Debillon, pour l'honneur que vous m'avait fait en acceptant de diriger mon travail, pour vos enseignements, vos conseils bienveillants, vos encouragements et votre amour communicatif de la néonatologie.

A Madame le Professeur P Hoffmann, pour l'honneur que vous me faites en participant à mon jury de thèse et en acceptant de juger mon travail.

A Madame le Docteur V Belin, pour l'honneur que tu me fais en participant à mon jury et pour tout ce que tu m'as appris autant médicalement qu'humainement sur nos quelques gardes. 
A mon père, consciemment ou inconsciemment responsable de ma vocation pour la pédiatrie et encore aujourd'hui source d'admiration pour ses connaissances abyssales.

Les gens disent «tel père, tel fils » et j'en suis fier.

A ma mère, qui m'a toujours fait confiance durant ma scolarité. Mille fois merci pour ton intendance parfaite de ma première année et pour ta bienveillance maternelle inébranlable depuis toujours.

A Laure, pour avoir été là du premier jour à l'amphithéâtre de propédeutique, en passant par le parc Chanot, jusqu'à Grenoble. L'internat fut une étape de plus avec toi en attendant les prochaines. Je n'y serais jamais arrivé sans toi.

A mon fils, ma plus belle réussite et mon bonheur au quotidien.

A ma sœur, que j'ai toujours su présente.

A Suzanne et Yannick, pour leur soutien et leur accueil chaleureux dans leur maison.

A tous mes amis et notamment les marseillais, ces six années avec vous furent un vrai bonheur.

A mes collègues internes de pédiatrie, avec une pensée particulière pour Anne, un petit bout de réanimatrice pédiatre qui gagne à être connue.

A Sébastien, pour ses multiples relectures, ses remarques toujours pertinentes et pour son humour au quotidien. On a bien rigolé.

A tous les médecins qui m'ont transmis leur savoir dans la bonne humeur et m'ont supporté pendant ces années. 


\section{Table des matières}

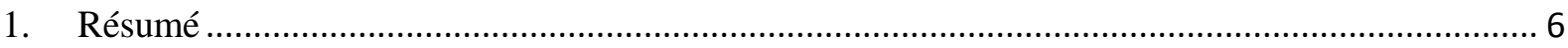

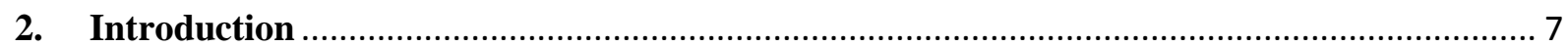



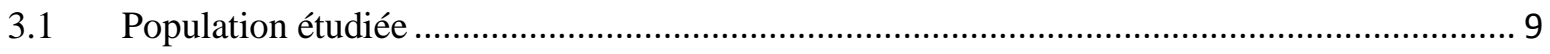

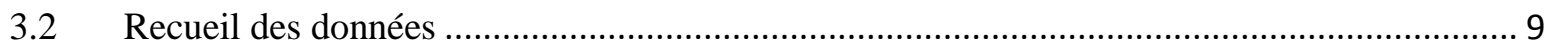

3.2.1 Caractéristiques de la grossesse et de l'accouchement................................................. 9

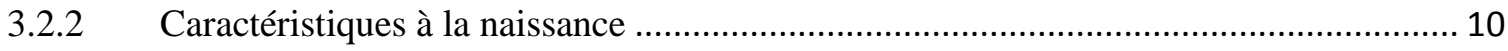

3.2.3 Caractéristiques pendant l'hospitalisation .................................................................. 10



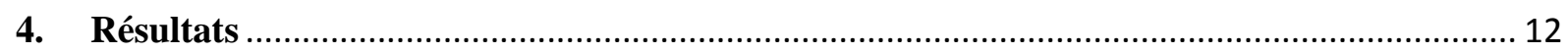

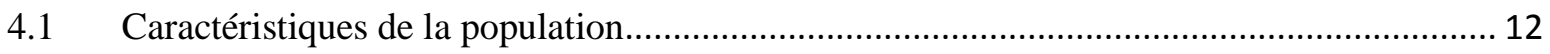



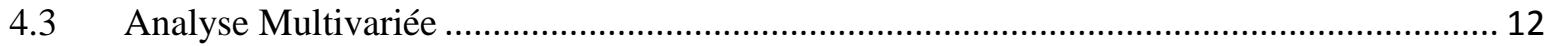

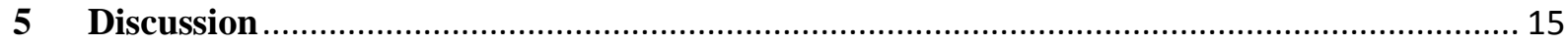

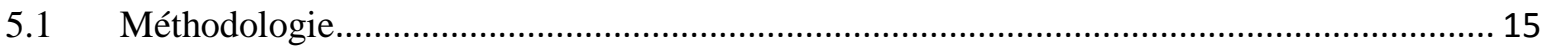

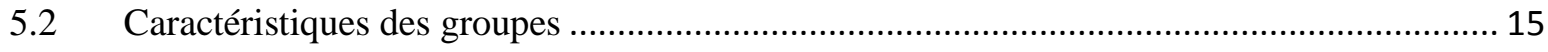

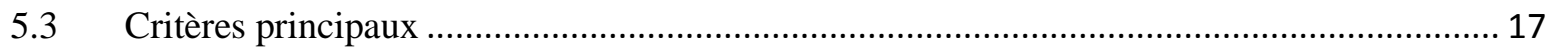



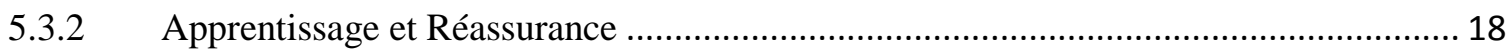

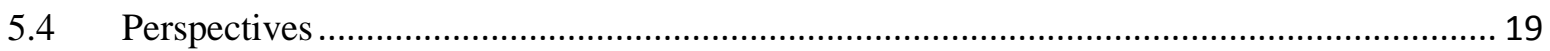



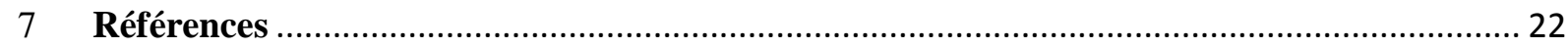




\section{Résumé}

Introduction: En France depuis les années 1990 de nouvelles unités de soins sont apparues au sein des maternités pour la prise en charge de la prématurité modérée, dites Unités MèreEnfant (UME).

L'objectif de notre étude était de comparer la durée d'hospitalisation entre l'UME et le service de Néonatologie des prématurés de 34 semaines d'aménorrhée (SA).

Matériel et méthodes: Il s'agit d'une étude rétrospective, monocentrique incluant 99 prématurés de 34 SA à 34 SA+ 6 jours hospitalisés entre 2004 et 2009 au CHU de Grenoble dont 31 en néonatologie (groupe NN) et 68 en UME (groupe UME). Ont été inclus tous les prématurés de $34 \mathrm{SA}$ hospitalisés en néonatologie ou en UME, les critères d'exclusion étaient la détresse respiratoire, les malformations congénitales, le poids de naissance inférieur à 1500 grammes, l'admission directe en réanimation néonatale et le transfert secondaire en UME.

Résultats : Les caractéristiques des deux groupes n'étaient pas significativement différentes en dehors du recours plus fréquent à une corticothérapie anténatale dans le groupe NN $84 \%$ contre $60 \%$ en UME ( $\mathrm{p}=0.04)$, d'un poids de naissance plus faible dans le groupe $\mathrm{NN}$ de $1892 \mathrm{~g}$ contre $2182 \mathrm{~g}$ en UME $(\mathrm{p}<0.01)$ et d'un âge gestationnel inférieur dans le groupe NN de 34.1 SA contre 34.3 SA en UME ( $\mathrm{p}<0.01)$. L'allaitement maternel exclusif à la sortie était significativement plus important dans le groupe UME $35 \%$ contre $13 \%$ en NN $(\mathrm{p}=0.04)$. Notre critère de jugement principal, durée d'hospitalisation, était significativement plus court en UME à 15.4 jours contre 20.7 jours en NN ( $\mathrm{p}<0.01)$ de même que la durée d'alimentation par sonde nasogastrique de 2,8 jours en UME contre 9,1 jours en NN $(\mathrm{p}<0.01)$. Cette différence demeurait après ajustement sur le poids de naissance et l'âge gestationnel.

Conclusion: Notre étude rétrospective montre que la durée d'hospitalisation des prématurés nés à 34 SA et hospitalisés au CHU de Grenoble est significativement plus courte lorsque ceux-ci sont admis en UME plutôt qu'en néonatologie. Pour cette raison, ce mode d'hospitalisation en UME en maternité peut être recommandé. 


\section{Introduction}

Le nombre d'enfants naissant prématurément n'a cessé de croitre ces dernières années passant de $9,4 \%$ à $12.3 \%$ des naissances soit $31 \%$ d'augmentation entre 1981 et 2003 [1]. Actuellement la population des prématurés nés à 34,35 et 36 semaines d'aménorrhée (SA) représente environ $75 \%$ des prématurés [2]. En parallèle de cette augmentation, de nouvelles unités de soins au sein même des maternités se sont crées avec pour but de prendre en charge ces enfants nés prématurément en dehors des structures classiques de néonatologie (NN). Plusieurs arguments sont énoncés comme bénéfices de ces unités mère-enfant (UME) :

- arguments psychologiques : pour éviter une séparation mère enfant [3] et ainsi réduire significativement le stress des parents et de l'enfant permettant un retour à la maison plus serein

- arguments médicaux : pour atténuer la gravité de certaines pathologies par la pratique du «peau à peau » permettant de stabiliser les enfants sur le plan thermique, respiratoire et digestif [4-5]

- arguments économiques : afin de diminuer le coût des hospitalisations par la réduction de temps d'hospitalisation [6-7]

Ces UME sont basées sur le concept de soins «kangourou », développé initialement en Amérique du Sud à l'institut maternel et infantile de Bogota en 1979. Cette méthode se définit comme une mise en peau à peau précoce, prolongée et continue d'un nouveau né hypotrophe, à terme ou prématuré, contre la poitrine de sa mère, que ce soit en milieu hospitalier ou à domicile et ce jusqu'à l'âge de 40 SA [8]. Elle a été initiée afin de pallier au manque de ressources matérielles dans les pays en voie de développement et pour permettre des sorties précoces [9]. En France, la première structure mère-enfant a été créée en 1987 à l'hôpital Antoine Béclère de Clamart et a été baptisée «Unité Kangourou ». Le concept était surtout d'éviter la séparation entre l'enfant et sa mère et de le soigner dans la chambre de la mère en déplaçant les soignants auprès de lui. Ce changement de pratique rentrait dans le plan quinquennal sur la périnatalité (1995-2000) lancé le 12 avril 1994 qui comprend 16 recommandations dont la douzième était de limiter la séparation «mère-enfant ».

L'UME du CHU de Grenoble a été créée en 2004 en collaboration avec les pédiatres de maternité, de néonatologie et les gynécologues obstétriciens. Elle comprend 6 lits intégrés à l'ensemble de l'unité de suites de couches. Le nouveau-né est pris en charge dans la chambre de sa mère d'où la nécessité de prolonger l'hospitalisation de la mère le temps de l'hospitalisation de l'enfant. Cette unité bénéficie d'un encadrement médical et paramédical mutualisé avec l'unité traditionnelle de suites de couches. Cet encadrement se compose de 4 sages-femmes et 6 auxiliaires le jour et 2 sages-femmes et 2 auxiliaires la nuit, 2 pédiatres et 2 internes en pédiatrie en journée. En 6 ans d'existence il y a eu 1178 nouveaux nés hospitalisés dont 153 prématurés de $34 \mathrm{SA}$ à $34 \mathrm{SA}+6$ jours. Les critères d'admission en UME sont la prématurité à partir de $34 \mathrm{SA}$, les hypotrophes à partir de 1500 grammes (g), les enfants de mères diabétiques sous insuline, les infections maternofœetales asymptomatiques, les difficultés transitoires d'alimentation. Les motifs d'hospitalisation les plus fréquents sont la prématurité et les infections maternofœetales.

L'objectif principal de ce travail était de comparer ces enfants nés prématurément hospitalisés en UME à ceux hospitalisés en néonatologie à propos de leur durée 
d'hospitalisation. L'hypothèse de travail est que les enfants bénéficiant d'une hospitalisation en UME ont une durée d'hospitalisation plus courte. Les objectifs secondaires étaient de comparer le délai de reprise du poids de naissance, la durée de l'alimentation entérale par sonde nasogastrique $(\mathrm{SNG})$ et la perte de poids maximale. 


\section{Matériels et méthodes}

\subsection{Population étudiée}

Il s'agit d'une étude rétrospective monocentrique sur la période de Janvier 2004 à Décembre 2009. Ont été inclus tous les prématurés de $34 \mathrm{SA}$ à $34 \mathrm{SA}+6$ jours hospitalisés en UME ou en NN. Ont été exclus les enfants hospitalisés en NN présentant des critères contre indiquant classiquement l'admission en UME: enfants présentant une détresse respiratoire, porteurs d'une malformation congénitale, ayant un poids de naissance inferieur à 1500 grammes. L'admission directe en réanimation néonatale et le transfert secondaire en UME étaient également des critères d'exclusion. La population a été divisée en 2 groupes correspondant au lieu d'hospitalisation : NN ou UME.

\subsection{Recueil des données}

Les données ont été recueillies rétrospectivement dans le dossier obstétrical et le dossier pédiatrique.

\subsubsection{Caractéristiques de la grossesse et de l'accouchement}

Age de la mère

Tabagisme maternel

Gestité et parité

Grossesses multiples

Diabète gestationnel

Pathologie maternelle: toxémie gravidique, hématome rétro placentaire, placenta prævia

L'étiologie de l'accouchement, noté idiopathique si aucune cause n'était retrouvée

La rupture de la poche des eaux (RPDE) supérieure à 18 heures

Le diagnostic d'infection maternelle probable est porté sur un faisceau d'arguments cliniques et paracliniques comprenant l'hyperthermie supérieure à $38^{\circ}$, l'élévation de la CRP et la positivité des prélèvements bactériologiques (examen cytobactériologique des urines, hémocultures, prélèvement vaginal) 
Prélèvement vaginal positif

Le mode d'accouchement : césarienne ou voie basse

Corticothérapie anténatale complète si la patiente a reçu 2 doses de Bétamethasone de $12 \mathrm{mg}$ et si la dernière précédait l'accouchement de plus de 12 heures.

\subsubsection{Caractéristiques à la naissance}

Le sexe

L’âge gestationnel en jours révolus

Le score d'Apgar à 1 et 5 minutes de vie

Le poids de naissance

La naissance Outborn

\subsubsection{Caractéristiques pendant l'hospitalisation}

Durée d'hospitalisation

Délai de reprise du poids de naissance

Délai d'ablation de la sonde nasogastrique

$\square$ La perte de poids maximale, exprimée en pourcentage du poids de naissance.

Le mode d'alimentation à la sortie de l'hôpital

Le recours à la photothérapie

L'utilisation d'une perfusion nutritive sans notion de durée

Utilisation d'un soluté de réhydratation orale (SRO), en sus de l'alimentation lactée, protocole de NN pour éviter le recours à une perfusion

$\square$ Une antibiothérapie supérieure à 5 jours

La survenue d'une hypoglycémie (glycémie capillaire < 2.2 mmol/1)

La survenue d'une hypocalcémie (calcémie sanguine $<2 \mathrm{mmol} / \mathrm{l}$ ) 


\subsection{Analyse statistique}

Les deux groupes ont été comparés sur chacune des caractéristiques. Pour les variables qualitatives nous avons utilisé le test de chi $^{2}$ ou le test exact de Fisher. Pour les variables quantitatives nous avons utilisé le t-test de Student Après cette analyse univariée, nous avons proposé une analyse multivariée basée sur un test d'ANOVA à variables multiples. Pour l'ensemble des tests le risque alpha a été fixé à 0.05 . Les calculs ont été réalisés à l'aide du logiciel Statview ${ }^{\circledR}(\mathrm{SAS} \circledast)$. 


\section{Résultats}

\subsection{Caractéristiques de la population}

Ce travail a inclus 99 enfants nés à 34 SA entre le 1er Janvier 2004 et le 31 Décembre 2009, dont 31 ont été hospitalisés en NN et 68 en UME. Le recueil des données a été complet sans données manquantes pour les deux groupes. L'ensemble des caractéristiques de la grossesse et de l'accouchement a été rapporté dans le tableau I. Il n'existe aucune différence statistiquement significative entre les données maternelles selon l'unité d'hospitalisation en dehors d'une corticothérapie complète plus importante dans le groupe UME. L'âge maternel moyen était de 29.7 ans en néonatologie et de 31.3 ans en UME, la grande majorité des mères étaient des multipares. On peut noter que plus de la moitié de ces accouchements prématurés étaient considérés comme idiopathiques. Une infection maternelle était suspectée dans 12.9 $\%$ des cas en néonatologie et dans $8.8 \%$ des cas en UME. Une césarienne a été pratiquée dans $54.8 \%$ des cas en Néonatologie et dans $39.7 \%$ des cas en UME..

L'ensemble des caractéristiques à la naissance et pendant l'hospitalisation est exposé dans le tableau II. Le caractère « outborn » était statiquement différent avec $29 \%$ des enfants nés outborn en NN contre aucun en UME $(p<0.01)$. L'âge gestationnel moyen était de 34.1 SA en Néonatologie et de 34.3 SA en UME $(\mathrm{p}<0.01)$. Le poids de naissance (PN) était également inférieur en Néonatologie, $1892 \mathrm{~g}$ contre $2182 \mathrm{~g}(\mathrm{p}<0$. 01). A la sortie l'allaitement maternel exclusif était plus important en UME (35.3\% vs $12.9 \%, p=0.04)$. Le recours à la photothérapie, la présence d'hypoglycémie, d'hypocalcémie et une antibiothérapie supérieure à 5 jours n'étaient pas statistiquement différents entre les 2 unités. L'utilisation en Néonatologie de perfusion $(\mathrm{p}<0.01)$ et du protocole SRO $(\mathrm{p}<0.01)$ était statistiquement différente car non utilisée en UME.

\subsection{Analyse Univariée}

Les objectifs en analyse univariée de notre étude sont exposés dans le tableau III. On trouve une durée de séjour et une durée d'alimentation par SNG significativement plus courte en UME par rapport à la NN. Cependant la perte de poids a tendance à être plus importante en UME et le délai de reprise du poids de naissance plus long sans toutefois atteindre la significativité statistique.

\subsection{Analyse Multivariée}

L'analyse univariée retrouve une durée plus courte d'hospitalisation en UME avec une population plus immature et de poids de naissance plus faible. Afin d'éliminer ces biais une analyse multivariée a été réalisée. Elle montre que la durée d'hospitalisation reste significativement plus courte dans le groupe UME $(p<0.005)$ après ajustement sur le poids de naissance et l'âge gestationnel. 


\begin{tabular}{|c|c|c|c|}
\hline & $\begin{array}{l}\text { Néonatologie } \\
(n=31)\end{array}$ & $\begin{array}{l}\text { Unité } \quad \text { Mère } \\
\text { Enfant } \\
(n=68)\end{array}$ & $\mathbf{p}$ \\
\hline Age maternel (année, moy $\pm \mathrm{DS}$ ) & $29,7( \pm 5.1)$ & $31,3( \pm 5.2)$ & 0,17 \\
\hline Tabagisme maternel & $19,3 \%(6)$ & $19,1 \%(13)$ & $>0,99$ \\
\hline Primipare & $19,4 \%(6)$ & $33,8 \%(23)$ & 0,22 \\
\hline Grossesse Multiple & $58,0 \%(18)$ & $48,5 \%(33)$ & 0,5 \\
\hline Diabète maternel & $16,1 \%(5)$ & $19,1 \%(13)$ & 0,94 \\
\hline Accouchement idiopathique & $51,6 \%(16)$ & $57,4 \%(39)$ & 0,75 \\
\hline Pathologie maternelle* & $19,3 \%(6)$ & $14,8 \%(10)$ & 0.78 \\
\hline $\mathrm{RPDE}>18 \mathrm{~h}$ & $22,6 \%(7)$ & $25 \%(17)$ & 0,99 \\
\hline Infection maternelle probable & $12,9 \%(4)$ & $8,8 \%(6)$ & 0,79 \\
\hline Prélèvements vaginaux positifs & $3,2 \%(1)$ & $13,2 \%(9)$ & 0,24 \\
\hline Césarienne & $54,8 \%(17)$ & $39,7 \%(27)$ & 0,23 \\
\hline Corticothérapie complète & $83,9 \%(26)$ & $60,3 \%(41)$ & 0,04 \\
\hline
\end{tabular}

Tableau I : Caractéristiques de la grossesse et de l'accouchement

* Pathologie maternelle : toxémie gravidique, hématome rétro placentaire, placenta prae 


\begin{tabular}{|c|c|c|c|}
\hline & $\begin{array}{l}\text { Néonatologie } \\
(n=31)\end{array}$ & $\begin{array}{l}\text { Unité } \quad \text { Mère } \\
\text { Enfant } \\
(n=68)\end{array}$ & p \\
\hline Garçon & $48,4 \%(15)$ & $57,4 \%(39)$ & 0,54 \\
\hline Apgar < 7 à $1 \mathrm{~min}$ & $6,5 \%(2)$ & $11,8 \%(8)$ & 0,65 \\
\hline Apgar $<7$ à $5 \mathrm{~min}$ & 0 & 0 & $\mathrm{NC}$ \\
\hline Naissance « outborn » & $29 \%(9)$ & 0 & $<0,01$ \\
\hline Age gestat. (SA, moy $\pm \mathrm{DS})$ & $34,1( \pm 0.3)$ & $34,3( \pm 0.3)$ & $<0,01$ \\
\hline PN $(g$, moy $\pm D S)$ & $1892( \pm 306)$ & $2182( \pm 260)$ & $<0,01$ \\
\hline Allaitement exclusif & $12,9 \%(4)$ & $35,3 \%(24)$ & 0,04 \\
\hline Photothérapie & $51,6 \%(16)$ & $52,9 \%(36)$ & $>0,99$ \\
\hline Protocole SRO & $22,6 \%(7)$ & 0 & $<0,01$ \\
\hline Perfusion & $29 \%(9)$ & 0 & $<0,01$ \\
\hline ATB $>5$ jours & $6,5 \%(2)$ & $2,9 \%(2)$ & 0,78 \\
\hline Hypoglycémie & $12,9 \%(4)$ & $8,8 \%(6)$ & 0,79 \\
\hline Hypocalcémie & $9,7 \%(3)$ & $27,9 \%(19)$ & 0,07 \\
\hline
\end{tabular}

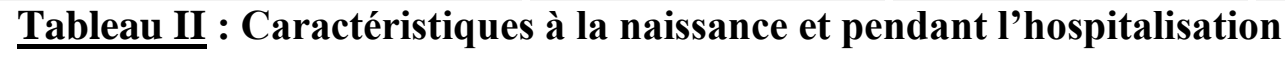

\begin{tabular}{|l|l|l|l|}
\hline & $\begin{array}{l}\text { Néonatologie } \\
(\mathbf{n = 3 1})\end{array}$ & $\begin{array}{l}\text { Unité } \\
\text { Enfant } \\
(\mathbf{n}=68)\end{array}$ & Mère \\
\hline Durée d'hospit. (jours, moy $\pm \mathrm{DS})$ & $20,7( \pm 5,6)$ & $15,4( \pm 3,8)$ & $\mathrm{p}<0,01$ \\
\hline Reprise PN (jours, moy $\pm \mathrm{DS})$ & $9( \pm 2,9)$ & $10( \pm 2,2)$ & $\mathrm{p}=0,07$ \\
\hline Durée de SNG (jours, moy $\pm \mathrm{DS})$ & $9,1( \pm 6,8)$ & $2,8( \pm 4,8)$ & $\mathrm{p}<0,01$ \\
\hline Perte de poids (moy $\pm \mathrm{DS})$ & $6,3 \%( \pm 3,1)$ & $7,4 \%( \pm 2,4)$ & $\mathrm{p}=0,08$ \\
\hline
\end{tabular}

Tableau III : Critères de jugement principal et secondaire 


\section{Discussion}

Notre étude retrouve une durée d'hospitalisation plus courte lorsque les prématurés de 34 SA à $34 \mathrm{SA}+6$ jours étaient hospitalisés en UME plutôt qu'en NN et ceci de façon indépendante du poids de naissance et de l'âge gestationnel. Des tendances non significatives sont montrées pour les enfants «UME » : la reprise du poids de naissance est plus longue pour ces enfants, avec une perte de poids initiale plus importante. Cependant plusieurs points sont à préciser :

\subsection{Méthodologie}

Il s'agit d'une étude rétrospective donc soumise à de multiples biais liés principalement à l'absence de randomisation de nos deux groupes d'études, entraînant un biais de recrutement. Par exemple le taux de corticothérapie est différent entre les 2 groupes, des tendances se dégagent également avec des mères plus souvent primipares en UME ou des césariennes plus fréquentes dans le groupe $\mathrm{NN}$.

En revanche notre recueil des données a été exhaustif avec des critères principaux peu sujets à l'interprétation comme la durée d'hospitalisation et d'alimentation entérale par sonde nasogastrique.

C'est une étude monocentrique. Elle reflète donc des pratiques spécifiques à une équipe obstétricale et pédiatrique au CHU de Grenoble.

Une des principales limites est l'effectif modéré de 99 enfants inclus, légèrement déséquilibré entre nos deux groupes. Cependant, cet effectif a permis de montrer une différence significative sur nos hypothèses principales et secondaires mais n'a pas permis de réaliser des tests suffisamment puissants pour les événements peu fréquents. Ceci incite à une prudence pour interpréter les résultats non significatifs. Ils peuvent témoigner d'une puissance insuffisante.

\subsection{Caractéristiques des groupes}

La question principale est la similitude de nos populations entre le groupe UME et le groupe NN. Les caractéristiques générales de la population ne mettent pas en évidence de différences significatives (hormis le taux de corticothérapie anténatale). En ce sens, nos populations sont donc comparables. Les résultats montrent néanmoins des différences non significatives sur le plan statistique, que nous allons commenter.

Les mères du groupe UME semblent plus souvent primipares, 34 \% en UME contre 19 $\%$ en NN (NS). On peut supposer que les femmes primipares, sans autre enfant à domicile, ont pu accepter l'hospitalisation en UME plus facilement, pouvant expliquer leur plus forte représentation dans ce groupe. Elles ont, a priori, moins de connaissances et d'expérience au niveau des soins de puériculture que les mères du groupe $\mathrm{NN}$ or la durée de séjour est plus courte dans le groupe UME. Cette inexpérience ne modifie donc pas la durée des hospitalisations. Ces mères «UME » ont tendance à être plus âgées, 31.3 ans contre 29.7 ans (NS). Ceci n'a probablement pas d'influence sur le critère de jugement principal. 
Le taux de césarienne serait plus important dans le groupe NN, $55 \%$ contre $40 \%$ (NS). On peut l'interpréter comme un biais de recrutement avec des enfants plus à risque dans la population NN. En effet les prématurés nés après césarienne ont des complications respiratoires plus fréquentes. Ceci n'a sans doute pas influencer la durée d'hospitalisation puisque les enfants présentant une pathologie respiratoire ont été exclus. La sur représentation des enfants nés par césarienne dans le groupe NN peut s'expliquer par une réticence des soignants à proposer l'UME après césarienne, du fait d'une moindre disponibilité maternelle. Pour toutes ces raisons le taux de césariennes supérieur dans le groupe NN ne semble pas modifier le critère «durée d'hospitalisation des prématurés » dans notre étude. Il pourrait surtout intervenir sur la durée d'hospitalisation de la mère.

Il existe une tendance à la positivité des prélèvements vaginaux dans le groupe UME de $13 \%$ contre $3 \%$ dans le groupe NN (NS) mais cette tendance n'est pas associée à un plus grand nombre d'infections maternofœtales. Le taux d'antibiothérapie du nouveau-né est non significativement différent entre les deux groupes. Il semble donc que cette tendance n'interfère pas dans la comparabilité des groupes d'autant plus que ces événements sont peu fréquents.

Le taux de corticothérapie est plus important dans le groupe NN, $84 \%$ contre $60 \%$ (p $=0.04)$ Cette différence doit s'interpréter comme un biais de recrutement de nos deux groupes. Les enfants ayant bénéficiés d'une corticothérapie sont sans doute issus de grossesses plus à risque, avec des mères peut être hospitalisées depuis plus longtemps ou ayant déjà fait une menace d'accouchement prématuré. Cependant la corticothérapie anténatale a montré ses bénéfices principalement sur le devenir respiratoire à court terme des prématurés [10-11]. Or nous avons exclu tous les nouveau-nés présentant une détresse respiratoire. Ainsi même si cette différence constitue un biais, elle n'a probablement pas ou peu d'influence sur notre critère de jugement principal puisque nos critères d'inclusion ont permis de la minimiser.

Le critère «naissance outborn » concernant $30 \%$ du groupe NN constitue une différence entre les deux groupes. L'absence d'outborn en UME est conforme aux indications d'hospitalisation dans cette unité puisqu'un nouveau-né ne peut y séjourner si la mère est absente. S'il existe une différence, il est difficile d'expliquer un lien entre celle-ci et le critère de jugement principal. L'influence du caractère inborn ou outborn sur le devenir immédiat du nouveau-né est d'ailleurs discuté. Arad et al n'ont pas établi de différence sur le devenir à court terme des prématurés nés inborn ou outborn sauf pour la mortalité immédiate [12].

Les enfants du groupe NN présentent un poids de naissance et un âge gestationnel significativement inférieurs à ceux du groupe UME, $1892 \mathrm{~g}$ contre $2182 \mathrm{~g}(\mathrm{p}<0.01)$ et 34.1 SA contre 34.3 SA $(p<0.01)$. Ces facteurs auraient pu constituer des biais importants, ce qui a justifié l'analyse multivariée. Celle-ci montre que la durée d'hospitalisation reste associée de façon indépendante à l'unité dans laquelle le nouveau-né se trouve $(\mathrm{p}=0.05)$.

Il convient également de noter que $28 \%$ des enfants du groupe UME présentent une hypocalcémie contre $9 \%$ dans le groupe $\mathrm{NN}(\mathrm{p}=0.07)$. A noter que ces hypocalcémies étaient toutes asymptomatiques. Cette tendance peut s'expliquer par un dépistage plus systématique en UME, avec des protocoles de surveillance sanguine moins formalisés en NN, du fait d'une surveillance accrue en unités de soins traditionnelles. Les apports calciques des enfants du groupe NN sont peut être mieux assurés puisque les enfants sont alimentés par SNG plus facilement si la tétée est insuffisante. Cependant, ces hypocalcémies ne semblent pas prolonger l'hospitalisation dans le groupe UME. 
Dans le même sens, la prescription de perfusion nutritive ou de SRO dans le groupe NN peut s'interpréter comme un biais de recrutement car si ces nouveau-nés nécessitent une perfusion c'est peut être qu'ils sont plus graves. Cependant le recours aux perfusions ou au SRO n'est possible qu'en NN. Les soignants l'utilisent peut être plus rapidement devant des difficultés alimentaires par manque de temps alors qu'une mère auprès de son enfant en aura forcement plus à lui accorder. On peut se poser la question si cette tendance est due à une différence entre nos groupes ou de pratique entre les unités. De plus une perfusion permettrait a priori une prise pondérale plus importante et indirectement une sortie plus rapide or ce n'est pas le cas dans notre étude.

Il est important de noter que notre groupe UME ne présente pas plus de complications durant leur hospitalisation avec un taux de survenue de photothérapie, d'hypoglycémie et d'antibiothérapie non statistiquement différent par rapport au groupe $\mathrm{NN}$, dans un cadre moins médicalisé qu'en service de Néonatologie classique. Par contre, nous n'avons pas pu évaluer le taux de réhospitalisation durant le premier mois après la sortie.

Le groupe UME bénéficie d'un allaitement maternel exclusif à la sortie plus important que le groupe $\mathrm{NN}, 35 \%$ contre $13 \%(\mathrm{p}=0.04)$, pouvant sans doute être un bénéfice de l'hospitalisation en UME du au maintien du couple mère-enfant. Cette présence permanente de la mère permet d'augmenter son temps de soins auprès de son enfant (mise au sein, gestes de puériculture) et de favoriser l'apprentissage et la réassurance parentale en vue de la sortie.

Nous observons donc des tendances exprimant des différences entre nos deux populations. Cependant, ces tendances ne semblent pas pouvoir expliquer à elles seules notre résultat principal de réduction de la durée d'hospitalisation en UME.

\subsection{Critères principaux}

Nous avons montré que dans notre étude la durée d'hospitalisation est plus courte lorsque les prématurés nés à 34 SA sont hospitalisés en UME plutôt qu'en NN. Il est probable qu'une des explications est le maintien d'un lien mère-enfant permanent. Plusieurs travaux ont bien établi qu'une hospitalisation en Néonatologie et donc une séparation de la mère et du nouveau-né perturbent la relation mère-enfant [3-13]. Nous allons discuter l'intérêt de maintenir cette relation sur plusieurs points :

\subsection{1 $\underline{\text { Temps de soins }}$}

L'hospitalisation en UME sous-entend l'hospitalisation de la mère auprès de son enfant. Ainsi la mère est en permanence auprès de lui permettant une adaptation optimale des soins au rythme du nouveau-né.

Il est surprenant de constater que dans notre étude les nouveaux nés du groupe UME ont une tendance à reprendre leur poids de naissance plus lentement que ceux du groupe $\mathrm{NN}$, 10 jours contre 9 jours $(\mathrm{p}=0.07)$ et ont une tendance à la perte de poids plus importante, $7 \%$ contre $6 \%(\mathrm{p}=0.08)$. On remarque également que le taux d'allaitement maternel exclusif 
est plus important dans le groupe UME que dans le groupe $\mathrm{NN}$ avec durée d'alimentation par SNG inférieure en UME qu'en NN, 2.8 jours contre 9.1 jours $(\mathrm{p}<0.01)$.

Ces résultats peuvent s'expliquer par le fait que les mères, étant en permanence avec leur enfant, ont plus de temps et donc une plus grande patience au niveau de l'apprentissage alimentaire. Alors que les enfants de NN sont sans doute plus rapidement mis en alimentation entérale sur SNG après un échec de tétée. De ce fait les nouveau-nés «UME» ont certainement un apport nutritif moindre dans les premiers jours de vie, correspondant à la mise en route de l'allaitement maternel. Ce délai se retrouve dans la perte de poids et la durée de reprise du poids de naissance plus important que dans le groupe NN. Cependant cela ne retarde pas leur sortie, on peut donc supposer qu'ils acquièrent une autonomie digestive plus précoce que ceux du groupe NN. On peut supposer un effet de la stimulation et de la persévérance maternelle à la prise alimentaire par tétée. Cette hypothèse est confortée par la durée moindre d'alimentation par SNG. Il semblerait que cette autonomisation digestive plus rapide des nouveau-nés UME joue un rôle important dans leur retour à domicile précoce.

Il est également possible qu'une approche moins technique, plus relationnelle de la prématurité favorise la mise en route d'un allaitement maternel efficace [14] dont les bénéfices sont prouvés par de nombreuses études [15].

\subsubsection{Apprentissage et Réassurance}

La séparation du couple mère-enfant entraine une augmentation des angoisses, déjà importantes du fait de la prématurité, autant du coté des parents [16] que du côté des prématurés [3]. Ces problèmes relationnels pourraient allonger la durée d'hospitalisation des enfants du groupe NN.

L'implication des parents dans les soins apportés à leur nouveau né est plus importante dans les UME du fait d'une présence continue de la mère. La participation aux soins des mères contribue à dédramatiser l'événement de la prématurité et l'abord médical des soins. La place de la mère auprès de son enfant est privilégiée et valorisée au maximum. L'enfant tire bénéfice des stimulations agréables qui lui sont apportées notamment du peau à peau. L'établissement du lien mère-enfant est favorisé, jouant sans doute un rôle dans la mise en place d'un attachement durable entre le nouveau-né et sa mère. Le but est de favoriser une autonomie plus rapide des parents dans les soins à apporter à leur enfant permettant un retour à domicile plus serein. [16]

Les critères de sorties sont peut être différents entre les unités. En UME le couple mère-enfant est au centre de l'évaluation et pas seulement le nouveau-né. Ainsi la décision de sortie implique sans doute beaucoup plus la mère qu'en $\mathrm{NN}$, avec une évaluation des compétences maternelles facilitées en UME du fait d'une présence permanente.

Il parait important de préciser que les équipes soignantes sont différentes dans les deux secteurs de soins étudiés avec des sages-femmes et des auxiliaires de puériculture en UME et des puéricultrices en néonatologie. Ces deux catégories professionnelles peuvent apprécier l'autonomie du nouveau-né avec des critères sensiblement différents. 
On peut supposer que ces sorties plus rapides d'UME sont notamment dues à un maintien et une valorisation du rôle parental ainsi qu'une responsabilisation précoce des parents dans les soins de leur enfant.

Cependant nous n'avons pas pu évaluer si ces séjours plus courts n'avaient pas des conséquences négatives sur la croissance, le développement psychomoteur et les ré hospitalisations des enfants.

\section{$5.4 \underline{\text { Perspectives }}$}

Comme nous l'avons dit les résultats de notre travail sont à mettre en balance par rapport à notre méthodologie.

Dans l'idéal, il faudrait réaliser une étude prospective, randomisée, multicentrique permettant d'obtenir deux groupes comparables et de se soustraire des pratiques propres à chaque centre.

Cependant il parait éthiquement difficile de randomiser les nouveau-nés entre les deux unités. Il faudrait donc se contenter d'une étude prospective et multicentrique. L'insu ( «aveugle ») ne serait pas possible lors de la prise en charge et durant l'hospitalisation. Les critères d'inclusion et d'exclusion seraient a priori à calquer sur les règles d'admission de l'UME, celles-ci étant probablement plus restrictives qu'en NN.

Par la suite, il faudrait réaliser une étude psychosociale sur le «vécu » des parents de l'hospitalisation, du retour à domicile et l'effet sur leur vie sociale de cette naissance prématurée.

Une visite médicale de l'enfant serait à prévoir à distance pour évaluer notamment sa croissance, son développement psychomoteur et relever les ré hospitalisations. Il serait intéressant de réaliser cette visite en insu du lieu d'hospitalisation de l'enfant.

Il faudrait enfin faire une étude économique avec évaluation du coût d'hospitalisation et du bénéfice ou du déficit sur le plan économique pour la structure d'accueil. En effet Charpak et al. [9, 17] estiment que les bénéfices économiques des sorties plus précoces des enfants d'UME sont effacés par le taux de réadmission hospitalière.

Une étude de ce type permettrait de conforter nos résultats sur la durée d'hospitalisation plus courte en UME et de définir les bénéfices ou non sur le ressenti parental et le coût économique. 


\section{Conclusion}

Notre étude rétrospective montre que la durée d'hospitalisation des prématurés nés à 34 SA et hospitalisés au CHU de Grenoble est significativement plus courte lorsque ceux-ci sont admis en unité mère-enfant plutôt qu'en néonatologie. Pour cette raison, ce mode d'hospitalisation en maternité peut être recommandé. Il conviendrait toutefois de vérifier que le taux de ré hospitalisations durant le premier mois n'est pas augmenté en cas d'hospitalisation en UME, ce que nous n'avons pas pu faire dans ce travail.

Notre conclusion ne peut toutefois pas être extrapolée à tous les prématurés ni à toutes les UME des différents centres hospitaliers. Afin d'évaluer plus largement le bénéfice des ces unités, il serait utile de valider cette étude rétrospective par une étude prospective et de compléter les données par une étude du vécu parental ainsi que du coût d'hospitalisation. 
THESE SOUTENUE PAR : CHABAUD François

TITRE : Influence du lieu d'hospitalisation sur le devenir à court terme des prématurés nés à 34 semaines d'aménorrhée.

\section{CONCLUSION}

Notre étude rétrospective montre que la durée d'hospitalisation des prématurés nés à 34 SA et hospitalisés au CHU de Grenoble est significativement plus courte lorsque ceux-ci sont admis en unité mère-enfant plutôt qu'en néonatologie. Pour cette raison, ce mode d'hospitalisation en maternité peut être recommandé. Il conviendrait toutefois de vérifier que le taux de ré hospitalisations durant le premier mois n'est pas augmenté en cas d'hospitalisation en UME, ce que nous n'avons pas pu faire dans ce travail.

Notre conclusion ne peut toutefois pas être extrapolée à tous les prématurés ni à toutes les UME des différents centres hospitaliers. Afin d'évaluer plus largement le bénéfice des ces unités, il serait utile de valider cette étude rétrospective par une étude prospective et de compléter les données par une étude du vécu parental ainsi que du coût d'hospitalisation.

VU ET PERMIS D'IMPRIMER

Grenoble, le $16 / 12 / 2010$

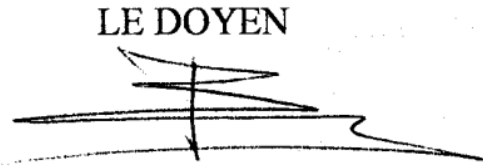

B.SELE
LE PRESIDENT DE THESE






\section{Références}

1. Davidoff MJ, Dias T, Damus K, et al. Changes in the gestational age distribution among U.S. singleton births: impact on rates of late preterm birth, 1992 to 2002. Semin Perinatol 2006, 30: 8-15.

2. Raju TN. Epidemiology of late preterm (near-term) births. Clin Perinatol 2006, 33: 751-763.

3. Christensson K, Cabrera T, Christensson E, et al. Separation distress call in the human neonate in the absence of maternal body contact. Acta Paediatr 1995, 84: 468-473.

4. Sizun J, Tran TA, et Lazartigues A. Peut-on améliorer le pronostic neuro-intellectuel et comportemental des enfants nés prématurément par une modification de leur environnement ? Arch Pédiatr 1999, 6: 7-11.

5. Sizun J, Ratynski N, Mambrini C. Implanter un programme individualisé de soutien du développement en reanimation neonatale: pourquoi, comment ? Arch Pédiatr 1999, 6: 434-439.

6. Charpak N, Ruiz-Pelaez JG, Figueroa de $\mathrm{C} \mathrm{Z}$ et al. A randomized, controlled trial of kangaroo mother care: results of follow-up at 1 year of corrected age. Pediatrics 2001, 108: 1072-1079.

7. Sloan NL, Camacho LW, Rojas EP et al. Kangaroo mother method: randomised controlled trial of an alternative method of care for stabilised low-birthweight infants. Maternidad Isidro Ayora Study Team. Lancet 1994, 344: 782-785.

8. Whitelaw A, Sleath K. Myth of the marsupial mother: home care of very low birth weight babies in Bogota, Colombia. Lancet 1985, 1: 1206-1208.

9. Charpak N, Ruiz-Peláez J G, Charpak Y. Rey-Martinez Kangaroo Mother Program: an alternative way of caring for low birth weight infants? One year mortality in a two cohort study," Pediatrics 1994, 94: 804-810.

10. Lacaze-Masmonteil T. Corticothérapie anténatale et accélération de la maturation fœtale. II. Résultats des applications cliniques. Arch Pédiatr 1996, 3: 1119-1128.

11. Stutchfield P, Whitaker R, Russell I. Antenatal betamethasone and incidence of neonatal respiratory distress after elective caesarean section: pragmatic randomised trial. BMJ 2005, 331: 662.

12. Arad I, Braunstein R, Bar-Oz B. Neonatal outcome of inborn and outborn extremely low birth weight infants: relevance of perinatal factors. IMAJ 2008, 10: 457-461.

13. Gaud G,Lapillonne A, Vachon H, et al. Même courte, l'hospitalisation en néonatologie perturbe la relation mère-enfant. Arch Pédiatr 1997, 4: 1153-1154.

14. Philipp B,Merewood A. The Baby-Friendly way: the best breastfeeding start. Pediatr Clin of North Am 2004, 51: 761-783.

15. Vohr BR, Poindexter BB, Dusick AM, et al. Beneficial effects of breast milk in the neonatal intensive care unit on the developmental outcome of extremely low birth weight infants at 18 months of age. Pediatrics 2006, 118: e115-123.

16. Bohu D, Mesbahi N, de Montgolfier-Aubron I, et al. Problèmes psychosociaux posés par le retour à domicile du nouveau-né prématuré. Arch Pédiatr 1999, 6: 264-266.

17. Charpak N, Ruiz-Pelaez JG, Figueroa de CZ, et al. Kangaroo mother versus traditional care for newborn infants $</=2000$ grams: a randomized, controlled trial. Pediatrics 1997, 100: 682-688. 